\title{
Exploring binding site of human RBP4 towards discovery of novel inhibitor for treatment of cardiovascular diseases
}

\section{Lakshmi Narayana Rao*, L. Raziya Beevi and Amineni Umamaheswari**}

Bioinformatics Centre, Department of Bioinformatics, SVIMS University, Tirupati. PIN - 517507.

*Presenting Author, ${ }^{* *}$ Corresponding Author, Email: svims.btisnet@nic.in

- Retinol Binding Protein 4 (RBP4) is a family of Lipocalin. It is the specific carrier of vitamin-A in plasma.

- Increased levels of serum RBP4 led to impaired glucose levels by liver, that leads to cardiovascular diseases.

\section{MMaterials and Rathods}

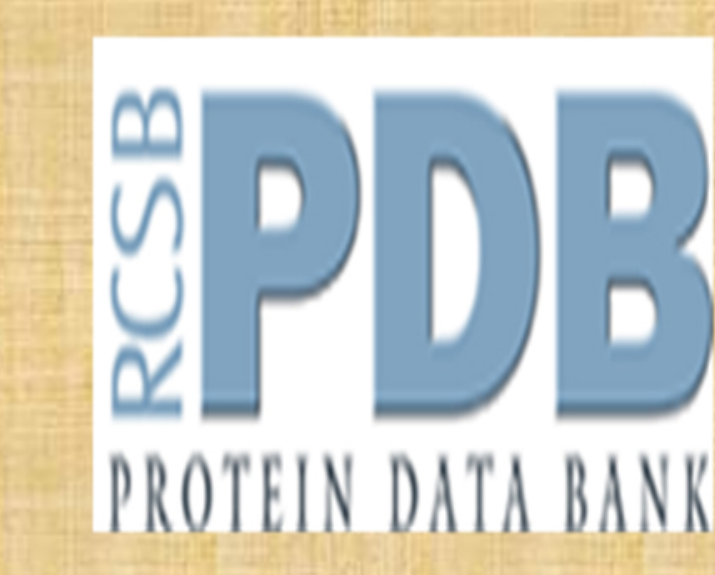

Retrieval of RBP4 structure

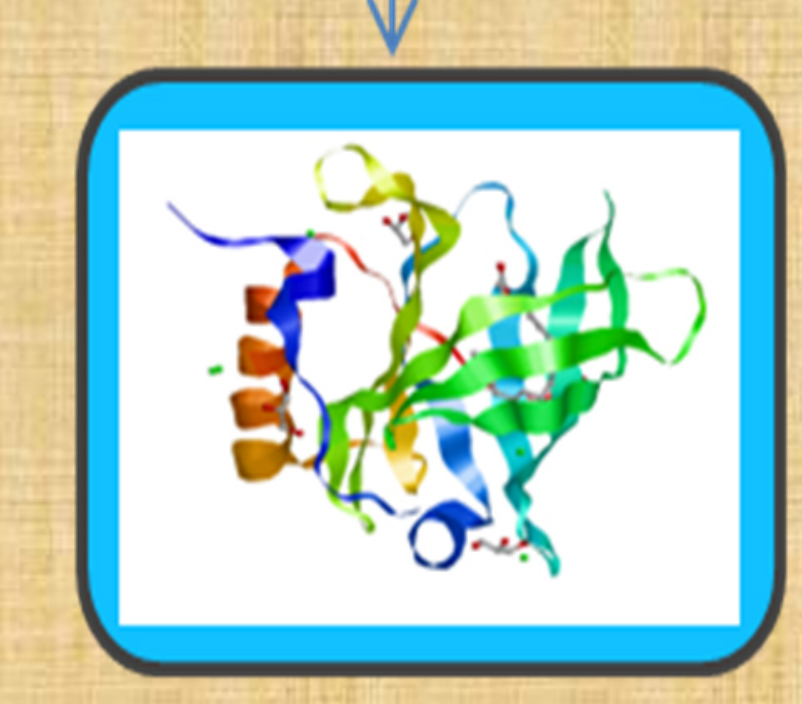

RBP4 protein structure

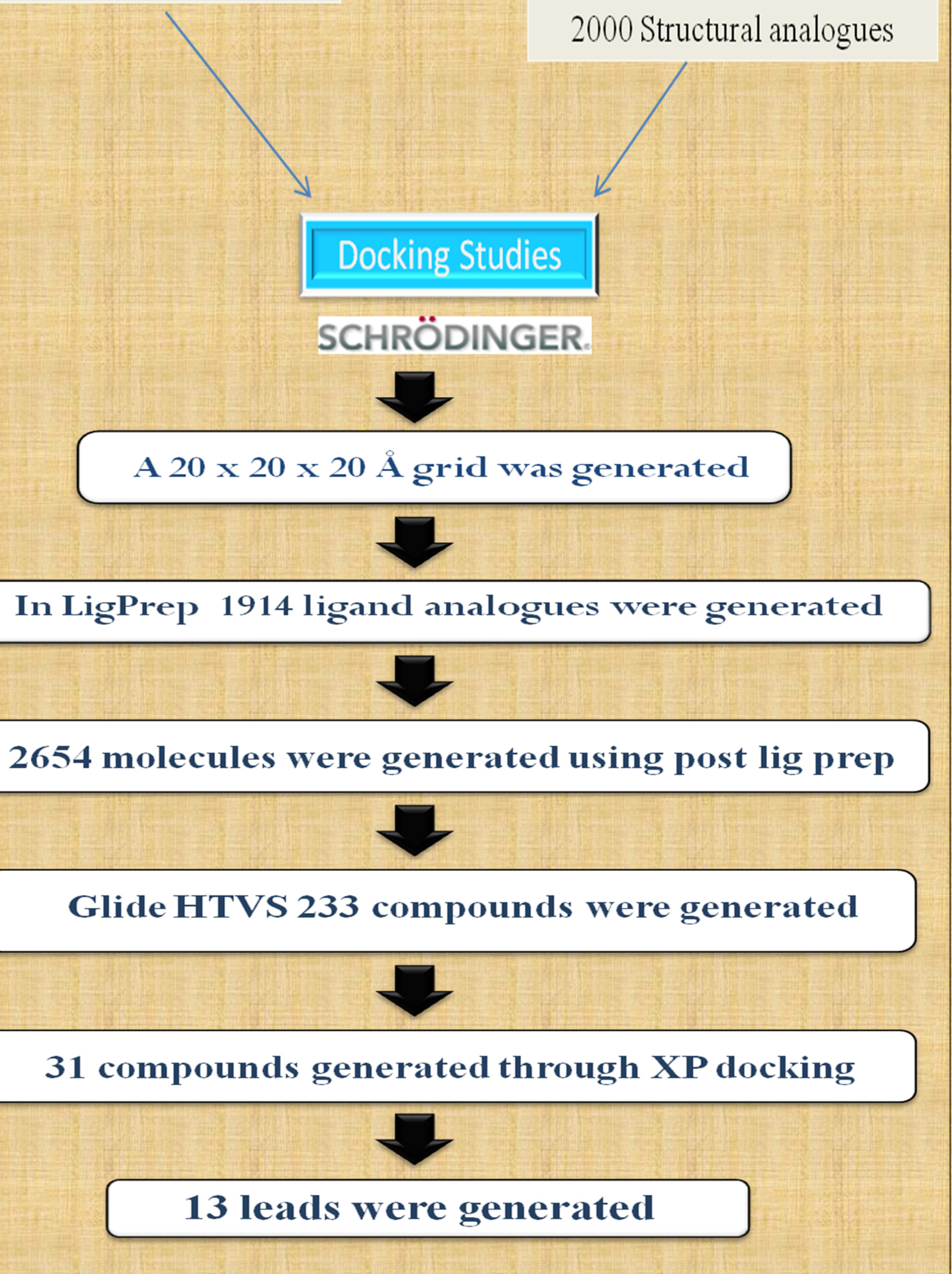

Five Published Inhibitors

Ligand.info

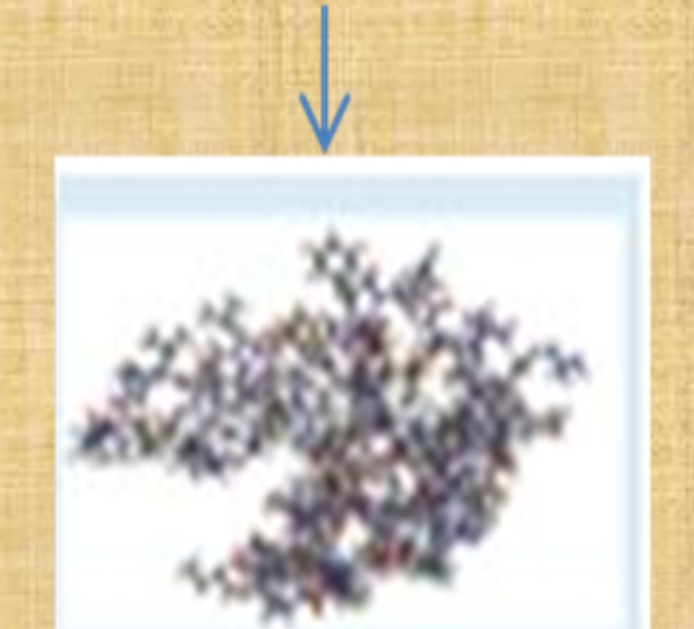

000 Structural analogues

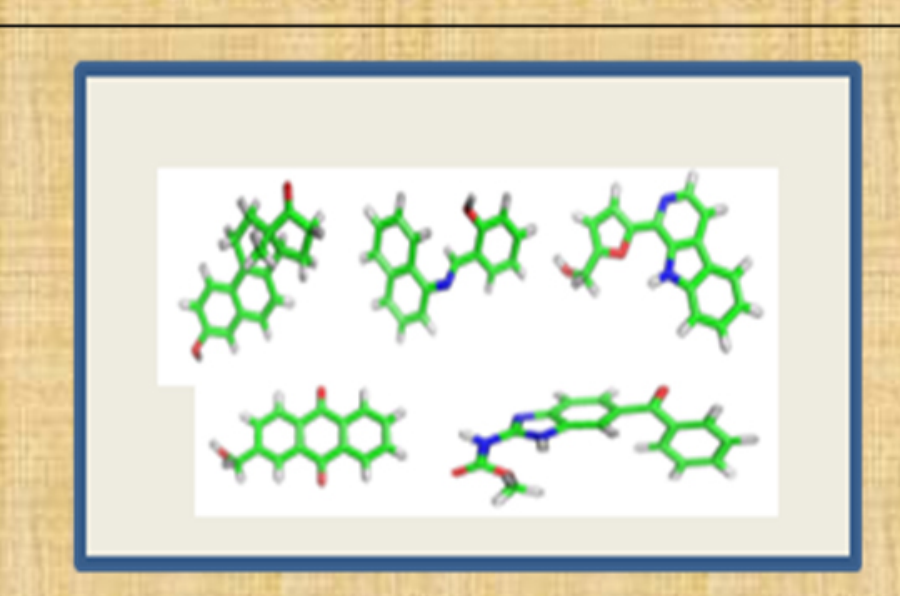

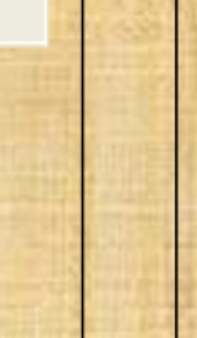$$
\text { . }
$$

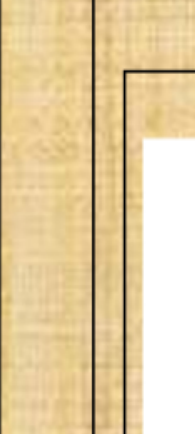
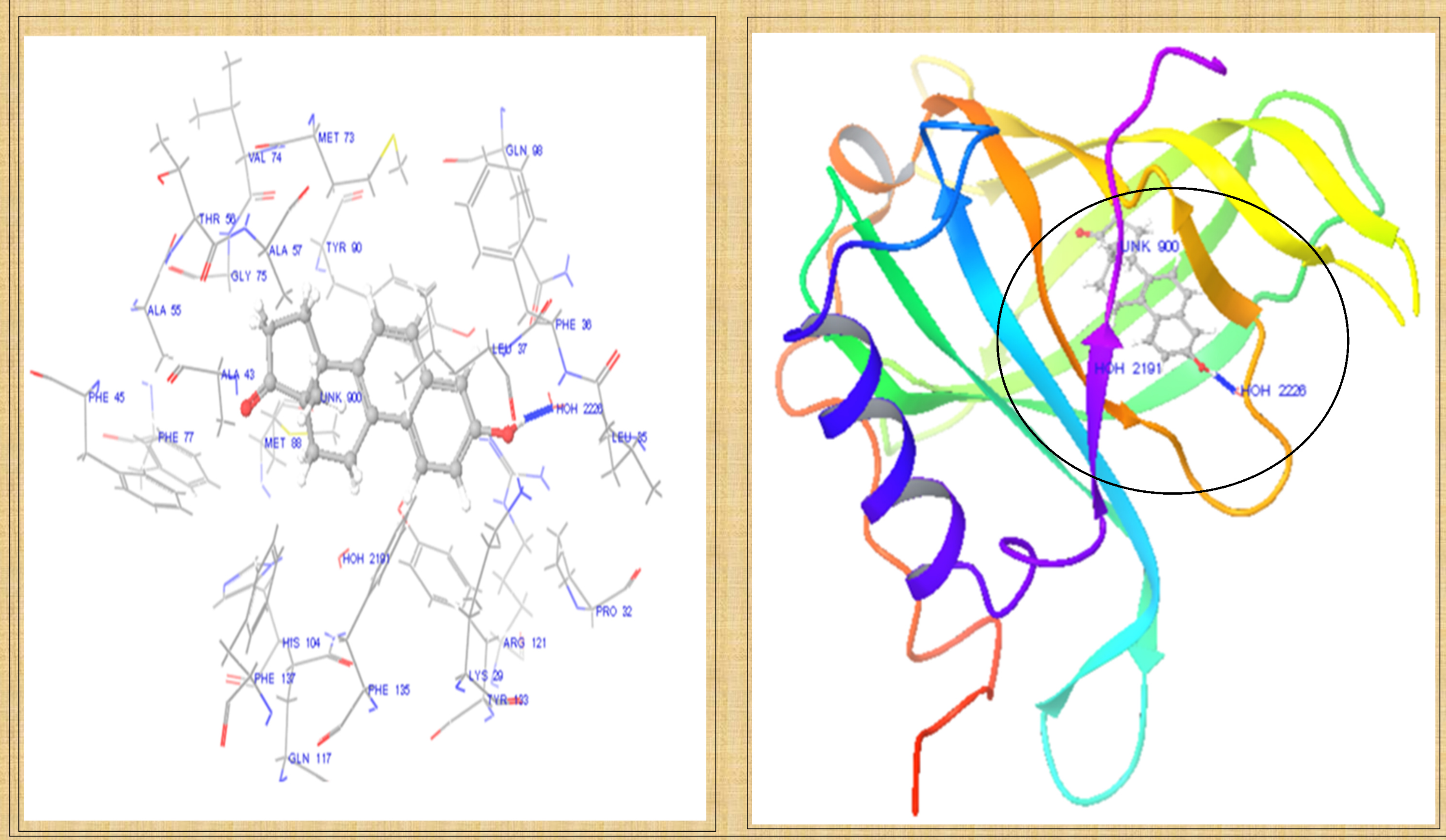

\section{Conclusion}

- Thirteen leads with good binding affinity to RBP4 were identified and compared with binding affinities and orientations of five published inhibitors.

- Lead '1' (equilenin) with lowest docking score $-12.874 \mathrm{~K} . c a l / m o l$ was reported as a best inhibitor against RBP4.

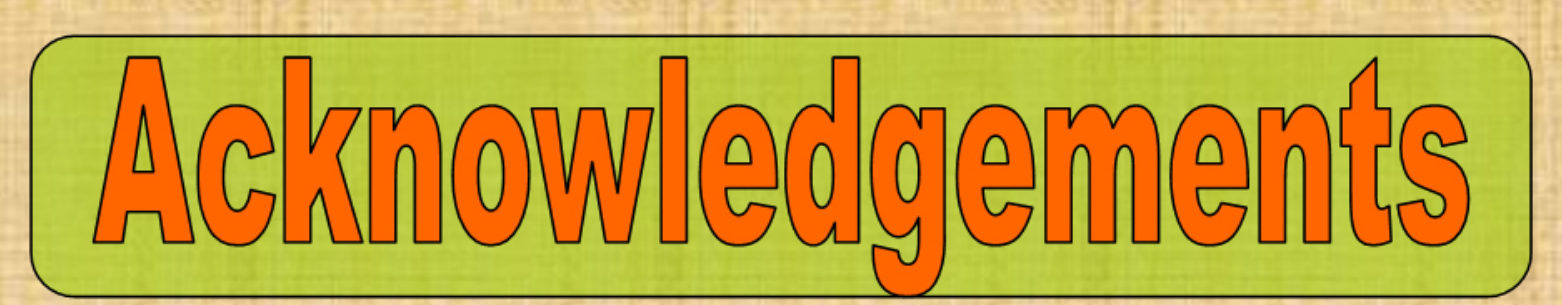

